\title{
The Stimulating Effect of Mineralized Bone Matrix on Articular Cartilage Regeneration
}

\author{
Yuri M. Iryanov, Olga V. Dyuryagina², Tatiana Yu. Karaseva ${ }^{3}$, Eugene A. Karasev ${ }^{3}$, \\ Nikolay A. Kiryanov** \\ ${ }^{1}$ Head of the Laboratory of Morphology, Russian Ilizarov Scientific Center Restorative Traumatology and Orthopaedics (RISC RTO), Kurgan, Russia \\ ${ }^{2}$ Laboratory of Purulent Osteology and Limb Defect Filling, Russian Ilizarov Scientific Center Restorative Traumatology and Orthopaedics (RISC RTO), Kurgan, \\ Russia \\ ${ }^{3}$ Department of Orthopaedics, Russian Ilizarov Scientific Center Restorative Traumatology and Orthopaedics (RISC RTO), Kurgan, Russia \\ ${ }^{4}$ Head of department of Pathology, Izhevsk State medical academy, Russia
}

Received: March 03, 2014; Accepted: April 27, 2015; Published: June 26, 2015

*Corresponding author: Kiryanov Nikolay A. Head of department of Pathology of Izhevsk State medical academy, Doctor of medical science, Tel: +7912-850 25 89, E-mail: kirnik@list.ru

\begin{abstract}
Background: The damage or loss of articular cartilage is costly medical problem. The purpose of this work is the morphological analysis of reparative chondrogenesis when implanted in the area of the knee joint cartilage of granulated mineral bone matrix.

Material and Methods: The characteristic features of the knee cartilage regeneration studied experimentally in pubertal Wistar rats after modeling a marginal perforated defect and implantation of granulated mineralized bone matrix obtained according to original technology without heat and demineralizing processing into the injury zone.
\end{abstract}

Results: This biomaterial established to have pronounced chondro- and osteo-inductive properties, and to provide prolonged activation of reparative process, accelerated organotypical remodeling and restoration of the articular cartilage injured.

Conclusion: The data obtained demonstrate the efficacy of MBM in clinical practice for the treatment of diseases and injuries of the articular cartilage.

2. Key words: Articular cartilage; Mineral component of bone matrix; Implantation; Reparative chondrogenesis.

\section{Introduction}

The damage or loss of the articular cartilage due to congenital anomalies, degenerative joint diseases or injuries has a negative effect on the quality of life of the injured in all age-related groups, and it is expensive to treat [1-3]. Surgical methods are often inadequate, and in many cases they do not give positive results in terms of proper replacement of articular hyaline cartilage [47]. In this regard, the development, scientific substantiation, and experimental morphologic evaluation of the biomaterials which have chondrogenic activity are of major importance in modern medicine. The most widely used biomaterials (demineralized bone matrix, matrices of polylactic and polyglycolic acids / PLA and PGA/, collagen cryogels, analogs of bone mineral, polysaccharides of natural origin) are characterized by complexity of manufacturing, low plastic effectiveness, the absence of chondrogenic activity, and limited biocompatibility [8-13] they are surrounded by fibrous capsule in organism. When they are prepared, the demineralizing substances are used which disorder natural microarchitectonics and chemical composition, thereby adversely impacting the integration properties and clinical results. Our previous studies have demonstrated that Mineralized Bone Matrix (MBM) obtained by using original technology without thermal and demineralizing processing [14] has osteo inductive properties, and it stimulates osteogenesis effectively [15]. Growth factors and bone morphogenetic proteins releasing during osteoclastic resorption established to be localized in MBM $[8,15]$, so it allowed us to assume that this biomaterial may possess stimulating properties in case of cartilage regeneration as well. We have not found such information in the available literature. The research is aimed at performing morphological analysis of reparative chondrogenesis when this biomaterial is implanted into the zone of the knee cartilage injury.

\section{Material and Methods}

All experiments in this study were performed in accordance with guidelines for animal research and were approved by our academy Ethics Committee.

\section{Experimental groups}

The experiments were conducted on 25 pubertal Wistar rats of 340-390 g weight (20 experimental and 5 intact ones). The process of keeping, experimenting, and euthanizing animals was regulated by the normative documents [16]. The operations on the animals were performed in an operating room under general anesthesia (8 $\mathrm{mg}$ of Rometarand $4 \mathrm{mg}$ of Zoletil per $1000 \mathrm{~g}$ of body weight intramuscularly). Non-through fenestrated defects of 2.5-3 mm in diameter were made on the patellar surface of the distal left and right femur with the dental drill, the subchondral bone was penetrated. Sterile granulated allogenic MBM (2-3 mg) 
obtained by original technology from tubular bones of mongrel rats after treatment in $6 \%$ sodium hypochlorite solution was placed into the right bone defect [14]. The defect in the left bone was left untreated under blood clot and it was used as control. The knee cartilage was studied in five intact normal rats for comparison. The animals were taken out of the experiment after $7,15,30$, and 60 days after the surgery (five animals were used for each time point).

\section{Morphological evaluation}

The pieces of femur bones of the operated rats and those of intact ones were fixed and embedded in paraffin (after decalcification) and in araldite (without decalcification). Paraffin sections were stained with hematoxylin-eosin, with picrofuchsin according to Van Gieson, and with Alcian blue (Alcian blue 8GS) at pH 2.5 and 1.0 to reveal non-sulphatedglycosaminoglycanes (NSGAG) and sulphated ones (SGAG), respectively [10]. The content of glycosaminoglycanes was determined in conventional units (c.u.) by defining Alcian blue concentration in sections according to the content of copper being a part of its composition (Alcian blue 8GS, $\mathrm{C}_{44} \mathrm{H}_{42} \mathrm{Cl}_{2} \mathrm{CuN}_{12} \mathrm{~S}_{2}$ ). We used INCA-200 Energy energy-dispersion X-ray spectrometer (X-ray electron probe microanalyzer) (Oxford instruments, England) [17]

The structure of MBM granules and regenerated bones in the injured area was investigated using JSM-840 scanning electron microscope and JEM-2010 transmission electron microscope (Jeol, Japan). The results of quantitative investigations were processed using the methods of variation statistics. The significance of the differences of the parameters compared was calculated using Student's t-test. The differences were considered significant at the level of $\mathrm{P}<0.05$.

\section{Results}

The implanted MBM granules are of 50-200 $\mu \mathrm{m}$ in diameter and have a well-ordered highly porous structure. The pores correspond to the places of localizing bone lacunae and bone tubules from which cells and other organic components have been removed when obtaining the biomaterial.

Seven days after the surgery, the joint capsule in both left and right limbs was hyperemic and edematic. The cartilaginous coating was dull. The signs of inflammatory reaction, and alternatively destructive changes affecting all metaepiphyseal components could be revealed around the injury. Foci of hematoma were found. They were infiltrated by fibrin clots, little-differentiated cell elements, neutrophilic granulocytes, macrophages, mast cells, extravasal erythrocytes, and lymphocytes. Leukocytic necrotic masses containing lysed cells and fibrin strata could be observed. Articular cartilage was scarified. The defect zone was partially replenished by granular and little-differentiated loose edematic connective tissue and few vessels. Sporadic bone-osteoid foci and isolated thin bone trabeculae were identified around the injury of the right bone (experiment). Reparative bone formation occured by the type of intramembranous osteogenesis. NSGAG and SGAG content in the zone of articular cartilage injury was reduced significantly comparing with the similar values in intact animals
( $0.59 \pm 0.03$, and $0.68 \pm 0.03$ c.u., respectively), however, there were no significant changes revealed in their content in control and experiment at this stage (Table 1).

Fifteen days after the surgery, numerous leukocytes, fibroblastic elements, bundles of collagen fibers, granulation tissue, and a significant number of dilated and filled with blood vessels could be identified in the defect central zone of control bone. Cellular-and-fibrillar elements of inflammation were not found in the right bone (experiment), reparative bone formation occured not only by the type of intra membranous osteogenesis, but by that of enchondral osteogenesis as well. In the zone of subchondral bone injury an extensive small-looped network was formed consisting of thickened trabeculae covered by rows of numerous large osteoblasts which produced spongy bone closely adhered with the surface of underlying bone. Implanted MBM granules resembled cystic cavities formed during decalcification of the sample and due to biodestruction. Functionally active osteoclasts with multiple nuclei and a brush-like border were located within these cavities (Figure 1). Numerous sinusoids were surrounded by proliferating peri-vasculocytes.

There were no hemorrhages and destruction foci in these areas, as well as active proliferation of fibroblasts, intense neoangiogenesis was observed. The layers of osteo- and chondrogenic cells at different differentiation stages were located on the granule surface. The zone of articular cartilage defect was partially filled with newly formed hyaline cartilage. Its intercellular substance contained NSGAG predominantly (Table 1). The defect edges merged with the edges of "maternal" articular cartilage where cell nests with isogenic groups were identified. The presence of the latters was evidenced by proliferative activity of some chondrocytes which were one of the sources of the cartilaginous tissue filling the defect. The cells located round MBM granules were the second source, and the chondrocytes in the zone of enchondralosteogenesis in the sites of subchondral bone fracture healing presented the third one. Glycosaminoglycane concentration in the defect zone for experiment more than twice exceeded control values (Table 1).

A significant part of bone defect was filled with loose or dense connective tissue 30 and 60 days after surgery in control (Figure $2, a, c)$. Newly formed cartilaginous tissue was formed only in the area of subchondral bone as a result of its injury, and it grew from the defect edges in the form of strip-like structures. In experiment the defect of articular cartilage was almost completely filled with newly formed hyaline cartilage having a smooth surface (Figure 2,b) which grew not only from the subchondral bone, but mainly from the defect edges where the isogenic groups of cells were located thereby, as evidenced by proliferative activity of chondrocytes.

The surface of newly formed hyaline cartilage acquired the shine characteristics of the articular cartilage of the intact animals. The cell population of the regenerated cartilage was mainly represented by proliferating chondrocytes, its structure did not possess the characteristics of the articular cartilage. Thirty days after surgery, the content of glycosaminoglycane 

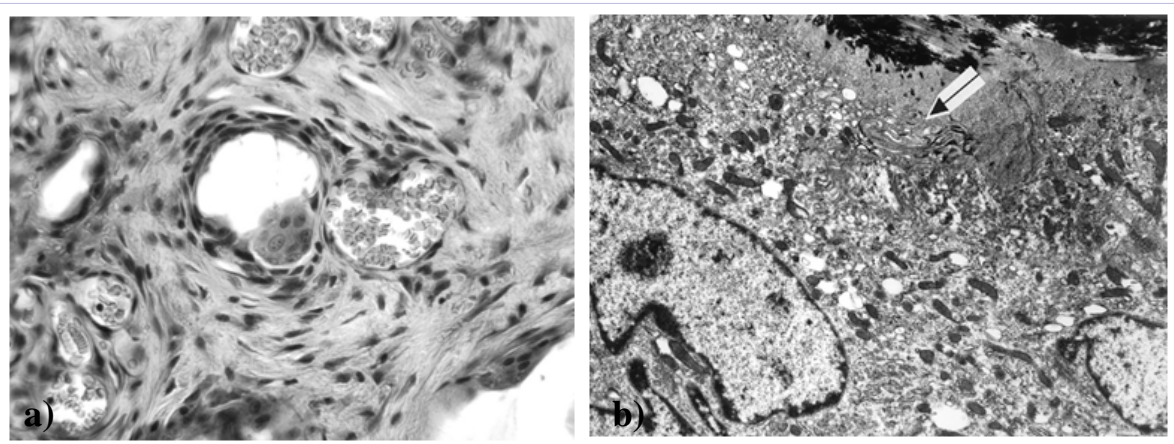

Figure 1: Zone of the defect of the rat knee cartilage 15 days after the surgery: a - an osteoclast within the implanted MBM granule which has the form of a cystic cavity, paraffin sections, staining with hematoxylin-eosin, magnification x 400; b - ultrastructure of the osteoclast localized on the surface of the implanted MBM granule, the arrow points to the osteoclast brush-like border in the zone of contact, ultrathin sections, transmission electron microscopy, magnification x 8000 .

Table 1: Glycosaminoglycane content in the zone of the knee cartilage injury in rats without defect plasty (control), and when using allogenic MBM (experiment) (M $\pm \mathrm{m}, \mathrm{c} . \mathrm{u}$.).

\begin{tabular}{|c|c|c|c|c|}
\hline \multirow{2}{*}{ The period of the experiment, days } & \multicolumn{2}{|c|}{ Control } & \multicolumn{2}{|c|}{ Experiment } \\
\cline { 2 - 5 } SGAG & NSGAG & SGAG & \multicolumn{2}{|c|}{ NSGAG } \\
\hline 7 & $0.18 \pm 0.01$ & $0.11 \pm 0.01$ & $0.20 \pm 0.01$ & $0.12 \pm 0.01$ \\
\hline 15 & $0.19 \pm 0.01$ & $0.12 \pm 0.01$ & $0.49 \pm 0.01^{*}$ & $0.26 \pm 0.01^{*}$ \\
\hline 30 & $0.21 \pm 0.01$ & $0.16 \pm 0.01$ & $0.56 \pm 0.02^{*}$ & $0.54 \pm 0.01^{*}$ \\
\hline 60 & $0.22 \pm 0.01$ & $0.24 \pm 0.01$ & $0.59 \pm 0.03^{*}$ & $0.70 \pm 0.01^{*}$ \\
\hline
\end{tabular}

* Significant changes comparing with control values.
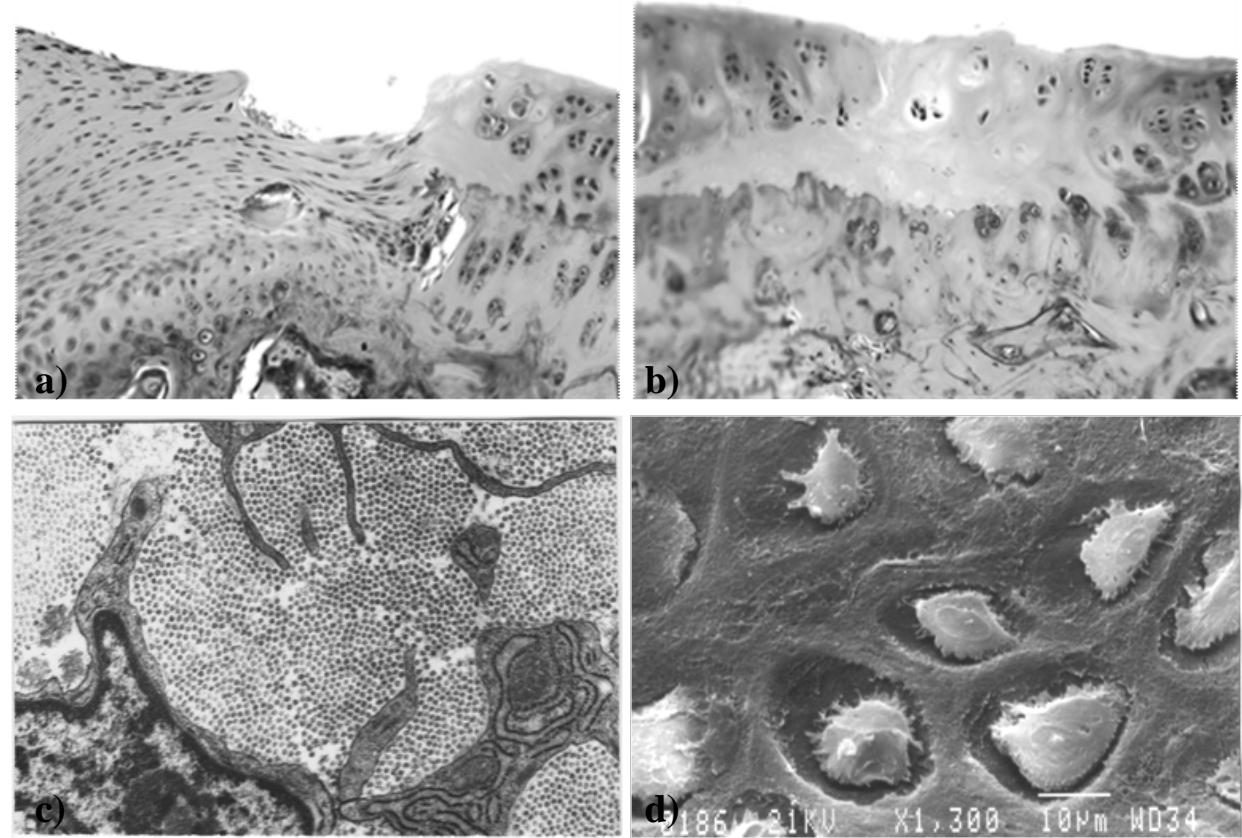

Figure 2: Zone of the defect of the rat knee cartilage 30 days after surgery: a, c - control, the defect is filled with loose fibrillar connective tissue; b, $\mathrm{d}$ - experiment, the defect is filled with newly formed hyaline cartilage; $\mathrm{a}, \mathrm{b}$ - paraffin sections, staining with Alcian blue at $\mathrm{pH} 2.5$, magnification $\mathrm{x}$ 200; c - fibroblasts and collagen fibril, ultrathin sections, transmission electron microscopy, magnification x 5000; $\mathrm{d}$ - chondrocytes are arranged as isogenic groups, scanning electron microscopy, magnification x 1300.

Citation: Iryanov YM, Dyuryagina OV, Karaseva TY, Karasev EA, Kiryanov NA (2015) The Stimulating Effect of Mineralized Bone 
non-suplphated and sulphated forms in the defect for experiment more than twice exceeded control values, but they did not differ from one another significantly. Sixty days after the surgery, glycosaminoglycanesulphated forms prevailed. It was evidenced by the higher maturity degree of the newly formed cartilaginous tissue (Table 1) which reached the maturity degree inherent for the articular cartilage of intact animals.

\section{Discussion}

Mature hyaline cartilage is known to have a slight potential of restoration due to low density of cells and little mitotic activity of chondrocytes [4,12]. Large defects of the articular cartilage are filled with biomechanically improper fibrillar cartilage followed by osteoarthritis development [5]. The advent of tissue engineering techniques has provided alternative possibilities to treat such patients by using cell therapy combined with synthetic substitutes of extracellular matrix and biologically active factors for functional replacement of articular hyaline cartilage $[1,11,18]$. The studies have demonstrated that the MBM granules when implanted into the defect zone of articular cartilage do not cause the immune response of rejection, and have a marked chondromodulating effect, as well as demonstrate prolonged activation of reparative chondro- and osteogenesis. They are observed in all the periods of further observation and gradually resorbed, only single MBM granules are found in the defect 60 days after surgery. Regenerated cartilage is formed early in the zone of articular cartilage injury, and it acquires the cell specificity of hyaline cartilage tissue. Integrated cartilage coating is formed, the disordered contours of articular surface are aligned gradually, thereby leading to complete or partial recovery of the joint functional activity. A sit can be assumed, that localized in the implanted MBM granules growth factors and bone morphogenetic proteins releasing during osteoclastic resorption provide chondro- and osteoinductor properties for the granules [8,19].

\section{Conclusion}

Thus, the studies have demonstrated that the use of the implant of granulated MBM as chondro- and osteogenesis stimulator and as a corrector of destructive disorders in bone and cartilaginous tissue in articular cartilage injuries seems theoretically justified and promising.

\section{Acknowledgements}

We thank the staff of our institutions for their help in carrying out experiments and supervision over animals during all stages of work.

\section{References}

1. Danisovic L, Varga I, Zamborsky R, Böhmer D. The tissue engineering of articular cartilage: cells, scaffolds and stimulating factors. Exp Biol Med (Maywood). 2012; 237(1):10-7. doi: 10.1258/ebm.2011.011229.

2. Guo Y, Yuan T, Xiao Z, Tang P, Xiao Y, Fan Y, et al. Hydrogels of collagen/chondroitin sulfate/hyaluronan interpenetrating polymer network for cartilage tissue engineering. J Mater Sci Mater Med. 2012; 23(9):2267-79. doi: 10.1007/s10856-012-4684-5.

3. Parmar PA, Chow LW, St-Pierre JP, Horejs CM, Peng YY, Werkmeister $\mathrm{JA}$, et al. Collagen-mimetic peptide-modifiable hydrogels for articular cartilage regeneration. Biomaterials. 2015; 54:213-25. doi: 10.1016/j. biomaterials.2015.02.079.

4. Brown TD, Johnston RC, Saltzman CL, Marsh JL, Buckwalter JA. Posttraumatic osteoarthritis: a first estimate of incidence, prevalence, and burden of disease. J Orthop Trauma. 2006; 20(10):739-44.

5. Buckwalter JA. Articular cartilage injuries. Clin Orthop Relat Res. 2002; (402):21-37.

6. Purcell BP, Lobb D, Charati MB, Dorsey SM, Wade RJ, Zellars KN, et al. Injectable and bioresponsive hydrogels for on-demand matrix metalloproteinase inhibition. Nat Mater. 2014; 13(6):653-61. doi: $10.1038 /$ nmat3922.

7. Steele JA, McCullen SD, Callanan A, Autefage H, Accardi MA, Dini D, et al. Combinatorial scaffold morphologies for zonal articular cartilage engineering. Acta Biomater. 2014; 10(5):2065-75. doi: 10.1016/j. actbio.2013.12.030

8. Deev RV, Isaev AA, KochishAIu, Tikhilov PM. Ways of development of cell technologies in bone surgery. TravmatolOrtopRossii. 2008; $47(1): 65-74$.

9. Lysenok LN. Biomaterials: contribution to the progress of modern medical technologies. KletochnTransplantolTkanInzheneriia. 2005; (2):56-61.

10. Microscopic technique. Eds. Sarkisov DS, PerovluL. Moscow. Medicine. 1996. $544 \mathrm{p}$.

11. Duguy N, Petite H, Arnaud E. Biomaterials and osseous regeneration. Ann Chir Plast Esthet. 2000; 45(3):364-76.

12. Strehl R, Tallheden T, Sjögren-Jansson E, Minuth WW, Lindahl A. Long-term maintenance of human articular cartilage in culture for biomaterial testing. Biomaterials. 2005; 26(22):4540-9.

13. Werkmeister JA, Ramshaw JA. Recombinant protein scaffolds for tissue engineering. Biomed Mater. 2012; 7(1):012002. doi: 10.1088/1748$6041 / 7 / 1 / 012002$

14.Ir'ianovluM, Ir'ianovaTlu. Patent RF. Biomaterial for filling bone defects and a technique to obtain it. No 2478394. Filed 23.11.2011. Publ. Bull 2013; (10).

15. Ir'ianovluM, Ir'ianovaTlu. Osteoplastic effectiveness of mineralized bone matrix. Morfologiia. 2013; 143(1):63-8.

16. European Convention for the Protection of Vertebrate Animals used for Experimental and other Scientific Purposes. VoprRekonstruktivPlastKhirurgii. 2003; (4):34-36.

17. Ir'ianovIuM, Ir'ianovaTIu. X-ray electron probe microanalysis in quantitative histochemistry. Morfologicheskievedomosti. 2010; (3):77-81

18. Eberli D, Atala A. Tissue engineering using adult stem cells. Methods Enzymol. 2006; 420:287-302.

19. Bessa PC, Casal M, Reis RL. Bone morphogenetic proteins in tissue engineering: the road from laboratory to clinic, part II (BMP delivery) J Tissue Eng Regen Med. 2008; 2(2-3):81-96. doi: 10.1002/term.74. 